\title{
Don't think, feel: basal ganglia-cortical connectivity underlies self- regulation of brain oscillations in humans
}

\author{
Kazumi Kasahara, ${ }^{1,2,3,4} \uparrow$ Charles S. DaSalla, ${ }^{1,2} \uparrow$ Manabu Honda, ${ }^{2}$ Takashi Hanakawa ${ }^{1,2,5} \dagger * *$ \\ ${ }^{1}$ Department of Advanced Neuroimaging, Integrative Brain Imaging Center, National \\ Center of Neurology and Psychiatry, Tokyo 187-8551, Japan. \\ ${ }^{2}$ Department of Functional Brain Research, National Center of Neurology and Psychiatry, \\ Tokyo 187-8551, Japan. \\ ${ }^{3}$ Human Informatics and Interaction Research Institute, National Institute of Advanced \\ Industrial Science and Technology, Ibaraki 305-8568, Japan. \\ ${ }^{4}$ FORESTO, Japan Science and Technology Agency, Saitama 332-0012, Japan. \\ ${ }^{5}$ Integrated Neuroanatomy and Neuroimaging, Kyoto University Graduate School of \\ Medicine, Kyoto 606-8501, Japan. \\ *Address correspondence to Takashi Hanakawa, hanakawa.takashi.2s@kyoto-u.ac.jp. \\ $\uparrow$ These authors contributed equally to this work.
}

\begin{abstract}
Brain-computer interfaces (BCIs) provide an artificial link by which the brain can directly interact with the environment. To achieve fine BCI control, participants must modulate the patterns of the cortical oscillations generated from the motor and somatosensory cortices. However, it remains unclear how humans regulate cortical oscillations, the controllability of which substantially varies across individuals. Here, we performed simultaneous electroencephalography (to assess BCI control) and functional magnetic resonance imaging (to measure brain activity) in healthy participants. Self-regulation of cortical oscillations induced activity in the basal ganglia-cortical network $(\mathrm{BgCN})$ and the neurofeedback control network (NfCN). Successful self-regulation correlated with striatal activity in the $\mathrm{BgCN}$, through which patterns of cortical oscillations were likely modulated. Moreover, BgCN and NfCN connectivity correlated with strong and weak self-regulation, respectively. The findings indicate that the $\mathrm{BgCN}$ is important for selfregulation, the understanding of which should help advance BCI technology.
\end{abstract}




\section{MAIN TEXT}

\section{Introduction}

Brain-computer interfaces (BCIs) provide an artificial link by which the brain can interact with the environment without using bodily effectors or sensors (1-4). In BCIs, signals from the brain are retrieved and decoded to control external devices. Neurofeedback (NFB) is a related technique which encourages users to control their own brain activity according to decoded brain signals (58). Potentials for real-world applications are emerging for both BCIs (9-13) and NFB (14-18), though these techniques have limitations. Brain networks of BCI/NFB users are engaged in finetuning their own neural states, involving self-regulation of brain activity or connectivity $(7,19$ 22). Furthermore, BCI/NFB performance varies across individuals, reflecting interindividual differences in the self-control of brain states (23-27). Some participants fail to self-regulate brain activity, even after repeated training sessions $(18,28,29)$.

Further development of BCI technology may overcome those limitations (30), but the issue will remain for NFB, which inherently relies on the ability to self-regulate brain activity (here, referred to simply as self-regulation). How organisms achieve self-regulation remains unknown, despite continued efforts to identify the underlying neural mechanisms and their correlates (18).

Self-regulation may involve the neurofeedback control network (NfCN), which includes the anterior insula cortex (AIC), anterior cingulate cortex, supplementary motor area (SMA), dorsolateral prefrontal cortex (dlPFC), lateral occipital cortex (LOC), and superior and inferior parietal lobules (SPL and IPL, respectively) $(14,16)$. The NfCN corresponds primarily to the cognitive control network (22), which has been implicated in top-down cognitive control. Thus, The NfCN might subserve the top-down control of self-regulation on the basis of explicit knowledge about the strategy (i.e., "think" strategy) (31). Alternatively, the basal ganglia-cortical network $(\mathrm{BgCN})$, which underlies behaviors stemming from trial-and-error-type procedural learning, may be the core neural correlate of BCI/NFB control $(32,33)$. Compelling evidence from rodents indicates that corticostriatal connectivity conveys essential information for BCIbased operant conditioning (21). Previous evidence points to the role of the $\mathrm{BgCN}$ in intuitive control of behaviors $(22,34)$, which may be called the "feel" strategy.

To investigate brain activity and connectivity during self-regulation, electroencephalography (EEG)-based BCI can be combined with functional magnetic resonance imaging (fMRI). Hinterberger et al. (33) conducted a pioneering concurrent BCI-MRI study with a sparse sampling method, revealing roles for both the $\mathrm{NfCN}$ and $\mathrm{BgCN}$ in self-regulation. More recently, a few concurrent BCI-fMRI studies reported the neural signature of motor imagery (32) and sense of control (35). These studies also indicated that cortical and subcortical regions are activated during a BCI-related task, but how the $\mathrm{NfCN}(18)$ and $\mathrm{BgCN}$ (21) jointly or distinctly contribute to self-regulation remains unknown. The $\mathrm{NfCN}$ and $\mathrm{BgCN}$ are not independent but, rather, are interconnected. Recent evidence indicates that part of the striatum may serve as a hub for different $\mathrm{BgCN}$ networks (37), requiring revision of the canonical theory of parallel and largely segregated $\mathrm{BgCN}$ circuits (36). Intriguingly, the connectivity of the striatum reflects individual variability in brain functions (38), which potentially accounts for the interindividual variability in self-regulation.

Here, we hypothesized that the "intuitive" $\mathrm{BgCN}$ and "logical" NfCN would play distinct roles in self-regulation during BCI. We also tested if the dorsal striatum serves as a hub interconnecting the $\mathrm{NfCN}$ and $\mathrm{BgCN}$. We show that the relative involvement of $\mathrm{NfCN}$ and $\mathrm{BgCN}$ in self-regulation reflects interindividual differences in BCI performance. 


\section{Results}

\section{BCI task}

Twenty-six healthy adults participated in the concurrent BCI-fMRI experiment. Each trial began with a presentation of a horizontal bar at the bottom of the screen indicating the left target (LT), the right target (RT), or a rest (Fig. 1A). A falling cursor was then displayed for $4 \mathrm{~s}$, during which its horizontal positioning was controlled by the laterality of the alpha-band $(9.5-12.5 \mathrm{~Hz})$ sensorimotor rhythms (SMRs) computed from electrodes C3 and C4 (23). The BCI task was to move the falling cursor horizontally to hit the target by modulating SMR laterality. For the RT and LT trials, participants were instructed to use first-person kinesthetic imagery of finger-thumb oppositions with the right and left hands, respectively. First-person kinesthetic imagery refers to a task by which the participants imagine themselves performing an action with an associated proprioceptive (not visual) sensation (39). The rest trials served as a perceptive control, during which participants were asked to pay attention to the falling cursor without performing motor imagery. After each trial, participants were briefly notified about whether the trial was a hit or a miss (outcome period).

\section{BCI performance}

The BCI experiment was performed over two sessions, including one practice session that was performed outside the MRI scanner while seated in a chair (outMRI) and one session that was completed inside the MRI scanner during concurrent BCI-fMRI acquisition (inMRI). After excluding data from two participants with excessive EEG artifacts, we evaluated the hit rates for the remaining 24 participants. Hit rates were calculated as the number of times the cursor hit an LT or RT divided by the total number of LT and RT trials. Twenty participants controlled the BCI significantly better than chance during the outMRI session, and 14 participants also did so during the inMRI session $(P<0.05$, two-tailed exact binomial test; see Table S1). The hit rates varied across participants during both the inMRI session (mean $=0.70 \pm 0.13$, range $=0.47-0.91)$ and outMRI session $($ mean $=0.60 \pm 0.09$, range $=0.45-0.80)$, but these were strongly correlated $(r=$ $0.83, P<0.001$; Fig. 1B). The offline analyses of the inMRI EEG data and extracted spectral amplitude $(2-23 \mathrm{~Hz}, 3-\mathrm{Hz}$ bins) showed that event-related desynchronization (ERD) of SMRs occurred mostly within the 11-Hz bin (9.5-12.5 Hz) (see Fig. S1 and Supplementary Text). The SMR ERD was contralateral to the BCI target and was correlated with BCI performance (RT: $r=$ $0.66, P<0.001$; LT: $r=-0.69, P<0.001)$. No statistical differences were found between the LT, $\mathrm{RT}$, and rest trials for any electrooculogram or electromyographic electrode channels (repeated measures ANOVA, $P>0.1$ for each channel), which indicates that BCI performance was not influenced by overt eye or body movements.

\section{Cortical and subcortical activity during BCI control}

We first examined whole-brain fMRI signal changes during the RT and LT trials (random-effects model analysis, $n=24 ; P<0.05$ cluster-level family-wise error [FWE] corrected). Compared with that during the rest trials, the self-regulation condition induced widespread activity involving both the $\mathrm{NfCN}$ and the $\mathrm{BgCN}$, including the bilateral premotor (PM)-SMA, SPL, IPL, dlPFC, AIC, visual areas, LOC, basal ganglia, thalamus, and posterolateral cerebellum (lobule VI) (Fig. 2 and see Table S2 for details). In particular, the primary motor cortex (M1), visual areas, and anteromedial cerebellum (lobule V) showed lateralized activity regarding the RT and LT tasks. Unexpectedly, ipsilateral M1 showed negative signal changes contributing to the SMR laterality, whereas contralateral M1 showed equivocal activity, which was not different from that of the rest trials. 


\section{Striatal activity and corticosubcortical connectivity supporting successful BCI control}

To clarify the mechanisms underlying self-regulation for BCI control, we explored activity throughout the brain correlated with hit trials. Only the bilateral posterior putamen demonstrated greater activity during hit trials than during miss trials $(t=3.48, P<0.05$ cluster-level $\mathrm{FWE}$ corrected; Fig. 3A). Conversely, cortical motor areas such as the SMA tended to show less activity during hit trials than during miss trials (Fig. 3A). We further examined whether this hitassociated activity of the posterior putamen was present during the BCI control period or during processing of information regarding the hit/miss outcome presented at the end of each trial. The ventral striatum, which is implicated in reward and motivational processing $(40,41)$, was chosen as a control striatal subsector. To assess the time course of brain activity, we identified volumes of interest (VOIs) in the dorsal putamen connecting with the motor cortices (motor putamen) and in the ventral striatum connecting with the orbitofrontal cortex, as defined by diffusion MRI (42). We found greater activity in the motor-associated putamen for hit trials than for miss trials for the control period (between target and outcome presentations) $\left(t_{(21)}=2.34, P=0.029\right.$, paired $t$ test), but not during the outcome period $\left(t_{(21)}=1.49, P=0.152\right)$ (Fig. 3B). By contrast, activity in the ventral striatum was comparable during the control and outcome periods. These findings suggest that motor striatal activity reflects brain mechanisms for self-regulation rather than for processing of outcome information related to a hit or miss.

These results also suggested that the motor striatum plays a role in successful selfregulation. However, it remained unclear how motor striatum activity influences BCI performance as determined by the laterality of the ERD with motor and somatosensory cortices (43). A possibility was that the BgCN modulates SMRs. To test this hypothesis, we performed a psychophysiological interaction (PPI) analysis, using the left motor striatum as the seed (Fig. 4A). When comparing hit and miss trials, the left motor striatum showed increased connectivity $(P<$ 0.05 FWE corrected) with the key nodes of the $\operatorname{BgCN}$ : the PM-SMA $(x, y, z=-2,8,58 ; Z=4.55)$ and globus pallidus $(x, y, z=-24,-12,2 ; Z=5.13)$ extending into the thalamus. Hit-related increased connectivity with the left motor striatum was also observed in the cerebellum $(x, y, z=$ $-38,-52,-40 ; Z=4.27)$ and visual cortex $(x, y, z=-12,-88,-6 ; Z=3.92)$. These results indicate the involvement of the $\mathrm{BgCN}(44-46)$ and, possibly, the cerebellar-basal ganglia circuit $(47,48)$ in successful modulation of SMRs. Furthermore, the laterality of ERD, the critical determinant of BCI performance, correlated with $\mathrm{BgCN}$ connectivity during the hit trials but not during the miss trials (Fig. 4B). These findings further corroborate that the motor striatum plays a pivotal role in the self-regulation of SMRs by modulating the connectivity within the $\mathrm{BgCN}$.

\section{Difference of functional brain networks related to individual performance}

Thus far, we found evidence for the role of the $\mathrm{BgCN}$, but not the $\mathrm{NfCN}$, in the self-regulation of SMRs. Building on previous studies on the neural mechanisms underlying BCI and NFB (23-26, 49-51), we exploited interindividual differences in self-regulation to test if the BgCN and NfCN jointly or distinctly contribute to successful self-regulation.

We examined whether effective connectivity with the hit-related motor striatum correlates with individual differences in BCI performance. In the PPI analysis at the individual level, the motor striatum showed various levels of effective connectivity with not only the $\mathrm{BgCN}$ but also the NfCN. The motor striatum regions of good performers tended to show connectivity with the $\mathrm{BgCN}$, while poor performers exhibited widespread striatal connectivity with the $\mathrm{BgCN}$ and the NfCN (Fig. 5A and B).

We quantified the extent to which motor putamen connectivity with the $\mathrm{BgCN}$ or NfCN correlates with individual differences in the hit rate, using least absolute shrinkage and selection operator (LASSO) regression analysis. The explanatory variables were the connectivity values between the left motor striatum and the key nodes of the $\mathrm{BgCN}$ and $\mathrm{NfCN}$ : M1, PM, SMA, thalamus, cerebellum, IPL, SPL, AIC, dlPFC, and LOC (see Table S2). This LASSO model 
predicted individual differences in BCI performance, with an $R^{2}$ of 0.87 . The left $\mathrm{PM}$ and right M1 showed positive weights, indicating stronger $\mathrm{BgCN}$ connectivity for good performers. Conversely, the right dIPFC, IPL, PM, and cerebellum as well as the left LOC and AIC showed negative weights, implicating poor performance for strong striatum-NfCN connectivity (Fig. 5C). These results indicated that strong striatum-BgCN connectivity coupled with weak striatum$\mathrm{NfCN}$ connectivity underlies the individual differences in self-regulation.

\section{Discussion}

We showed that activity and connectivity of the $\mathrm{BgCN}$ reflects controllability of a $\mathrm{BCI}$ at both within-individual (hit vs. miss) and interindividual levels, providing evidence that $\mathrm{BgCN}$ supports the self-regulation of SMRs. Rather unexpectedly, the recruitment of the NfCN, which might reflect attempted top-down control over a BCI/NFB task (18), had a detrimental impact on selfregulation.

\section{Neural activation and deactivation during BCI control}

Consistent with previous work $(32,35)$, the BCI task used in this study recruited motor-related cortical and subcortical areas. Contralateral M1 activity did not differ between the task and rest periods (see Fig. 2); this finding agrees with previous studies showing equivocal M1 activity during motor imagery $(52,53)$. However, our finding of a decrease in ipsilateral M1 activity during BCI seems novel and requires some explanation. During unilateral hand movement, ipsilateral M1 activity can be suppressed below a resting baseline (54). This phenomenon is usually interpreted as the manifestation of interhemispheric inhibition. However, the concept of interhemispheric inhibition does not explain the present finding, because ipsilateral M1 activity decreased without increases in contralateral M1 activity. We propose that, in addition to the increase in neural/synaptic activity ("activation"), suppression of activity below that seen at the rest baseline (i.e., "deactivation") also contributes to BCI task control. This interpretation is based on the following considerations. Synchronized SMRs are a signature of a deactivated or "idling" motor cortex (43). Thus, a downregulation of ipsilateral motor area activity to levels lower than those during the rest periods should correlate with ipsilateral SMR synchronization. In our study, increasingly synchronized SMRs in the ipsilateral motor cortex enhanced ERD laterality, yielding better BCI control (Fig. S1c). Moreover, ipsilateral M1 connectivity with the striatum correlated with the individual differences in self-regulation, supporting the effectiveness of the ipsilateral "deactivation" strategy through the BgCN. This hypothesis should be tested in future work, since the downregulation of brain activity below a given baseline is considered an idiosyncratic strategy for BCI control (22).

\section{Striatal activity and goal-directed modulation of brain rhythms via the $\mathrm{BgCN}$}

A key finding from the present study is that motor striatal activity correlated with BCI performance. Striatal activity might reflect processing of the outcome stimuli, corresponding to "consummatory" processes during the receipt of a reward $(40,41)$. Although we did not use explicit incentives such as monetary rewards, striatal activity can be elicited merely by positive reinforcement (55). However, we considered this consummatory motor striatal activity unlikely, because hit-related motor striatal activity occurred during the BCI control period but not during the outcome period. Moreover, the ventral striatum, which underlies consummatory behavior (41), exhibited robust BCI task-related activity irrespective of the outcome. This indicates that the ventral striatum supports the motivation necessary to complete a BCI task (56) regardless of whether the response is a hit or miss.

Current BCIs often require a learning period before users can achieve adequate control $(22,57,58)$. In the present study, BCI control did not improve over the short experimental period during fMRI; thus the hit-related striatal activity cannot be ascribed to BCI learning. Still, we 
consider that the striatal activity might relate to behaviors categorized as implicit and unsupervised learning based on trial-and-error experiences $(31,59)$. Indeed, previous studies found striatal activity during intuitive mental processes for trials and errors $(33,60,61)$. Therefore, the motor striatum may serve self-regulation through an intuitive "feel" type of strategy.

Increased striatal activity during the hit trials was accompanied by increased effective connectivity with important nodes of the $\mathrm{BgCN}$ (Fig. 4A). The $\mathrm{BgCN}$ constitutes a semiclosed loop implicated in a variety of functions, including behavioral selection and switch, procedural learning, and motor and cognitive vigor $(44,45)$. The striatum may switch relevant networks according to the behavior $(62,63)$. We also found that $\mathrm{BgCN}$ connectivity correlated with ERD laterality, which was the critical determinant of BCI performance (Fig. 4B). This finding provides new evidence that the striatum is involved in modulating brain rhythms through the $\mathrm{BgCN}$. Therefore, the striatum needs to be included as an important module of the classic circuit for the generation of SMRs: the thalamocortical circuit $(64,65)$. The goal-directed modulation of SMRs may relate to the striatum's role in creating a response bias in the cerebral cortex during demanding tasks $(46,66)$.

During the hit trials, the motor striatum also showed increased effective connectivity with the cerebellum, which also exhibited substantial BCI task-related activity. This suggests that the basal ganglia interacts with the cerebellum for successful BCI control. Anatomical evidence indicates direct and reciprocal cerebellar-basal ganglia circuits via the thalamus $(47,48)$, and these circuits may thus contribute to BCI control via the thalamic region, as revealed by the PPI analysis.

\section{BgCN vs. NfCN}

The present results demonstrate that the $\mathrm{BgCN}$ and $\mathrm{NfCN}$ play different roles in self-regulation. Good BCI performers exhibited strong striatal connectivity with the $\mathrm{BgCN}$, which included regions that subserve an implicit or "bottom-up" strategy of behavioral control $(31,59)$. BCI control may require striatal functions to modulate activity in the cortical areas to lateralize ERD. By contrast, the LASSO regression analysis revealed that $\mathrm{NfCN}$ connectivity was detrimental for BCI performance. Poor BCI performers exhibited stronger connectivity with the NfCN, including the dlPFC, IPL, and LOC. This suggests that the use of an effortful "top-down" or cognitive strategy results in poor BCI performance. In other words, subjects who exhibited poor BCI control might have adapted a "think" strategy to control SMRs declaratively or explicitly according to the task instructions about motor imagery. The improvement in BCI performance through training may involve shifts in which neural substrates are recruited, from those underlying cognitively demanding control (NfCN: "think") to those related more to intuitive (34) and automatic (22) control (BgCN: "feel"). The employment of these two strategies at an early stage of learning may explain the interindividual differences observed in this study.

The present findings indicate that it is better to "feel" than to "think" to modulate SMRs as a BCI control signal. Researchers should thus tell BCI experiment participants "Don't think. Feel! Don't concentrate on the target but concentrate on the feeling from the fingers." Our findings indicate that altering the instructions given to participants will promote the implementation of an effective strategy and thus reduce interindividual differences in BCI controllability. 


\section{Materials and Methods}

\section{Experimental Design}

\section{Participants}

Twenty-six healthy participants (12 female; mean age \pm standard deviation [SD], $22.4 \pm 2.9$ years) participated in this study. Each participant performed two outMRI runs and three inMRI runs, which were completed on different days. All participants took part in an outMRI study before the inMRI study (23). All participants were right handed, as assessed using the Edinburgh Handedness Inventory (68), had normal or corrected-to-normal vision, reported no history of neurological or psychological disorders, and had no prior BCI experience. Written informed consent was obtained from all participants before participation, according to the study protocol that was approved by the institutional review board of the National Center of Neurology and Psychiatry, Tokyo, Japan. After visual inspection, data from two participants were discarded due to excessive movement-related EEG artifacts during fMRI; hence, data from 24 participants were analyzed.

\section{Simultaneous EEG-fMRI acquisition}

EEG was used as a BCI control modality because of its wide application in the field of neuroprosthetic control and neurorehabilitation (9-11). A prototypical BCI was employed that uses SMRs, which arise from M1 and somatosensory cortices $(32,33,35,43)$. fMRI was used to measure neural/synaptic activity throughout the brain, including $\mathrm{NfCN}$ and the BgCN. Blood oxygen level-dependent (BOLD) fMRI acquisition was performed with a 3-T MRI scanner (Magnetom Trio; Siemens, Erlangen, Germany) using a T2*-weighted, gradient echo, echo planar imaging sequence (repetition time, $3 \mathrm{~s}$; echo time, $30 \mathrm{~ms}$; flip angle, $90^{\circ}$; voxel size, $3.0 \mathrm{~mm}^{3}$; number of slices, 42). A total of 262 scans were acquired for each run. The first 45 scans were dummy scans to minimize the transient effects of magnetic saturation and to initialize the artifact correction and BCI classifier algorithms.

Electrophysiological data were simultaneously acquired using MRI-compatible amplifiers (BrainAmp MR plus; Brain Products, Gilching, Germany) and a customized EEG cap (BrainCap MR; Brain Products) (69). The EEG cap consisted of 13 electrodes; nine were positioned over the sensorimotor area (F3, F4, C3, C4, Cz, P3, P4, T7, and T8), one over the left eye (Fp1), one as the ground electrode $(\mathrm{AFz})$, one as the reference electrode $(\mathrm{FCz})$, and one attached by a $35-\mathrm{cm}$ lead and placed on the back to record the electrocardiogram. Total impedances were kept at $<15 \mathrm{k} \Omega$. Electromyograms over the left and right thenar muscles and horizontal electrooculograms were also simultaneously acquired. Data were sampled at 5,000 Hz and filtered with $0.1-\mathrm{Hz}$ high-pass and $250-\mathrm{Hz}$ low-pass hardware filters.

\section{Online EEG artifact correction}

To provide online BCI classification and online feedback, MRI artifacts incurred on the EEG data were corrected online. According to methods reported by Allen and colleagues (70), artifact correction algorithms were written in MATLAB R2007b (MathWorks, Natick, MA, USA) that operated in conjunction with the BCI software. The system first corrected gradient artifacts, which are millivolt-scale distortions in EEG that are caused by the switching gradient magnetic fields of MRI. Exploiting the fact that gradient artifacts are mostly stationary and phase locked to the repetition timing of a sequence, mean gradient artifact templates were calculated for each channel and subtracted from the incoming EEG.

After gradient artifact correction, the system then corrected for ballistocardiogram artifacts, which are microvolt-scale deflections in the EEG resulting from micromovements of the head that are induced by the pulsatile acceleration of blood through the aortic arch (71) and 
possibly also by the expansion and contraction of scalp arteries (70). The R peak of electrocardiogram precedes artifacts by approximately $200 \mathrm{~ms}$; this information was used to create a mean ballistocardiogram template over the R-R interval for each channel, which was subtracted from the EEG, similarly to the gradient artifact correction process.

To limit the effects of gross and ballistocardiogram-related head movements, a custommade vacuum cushion (72) was placed around the participant's head that conformed to the space inside the MRI head coil. Finally, to reduce remnant scanner-related noise and baseline drift, a 12th-order elliptic bandpass filter (1-23-Hz bandpass, 0.1-dB passband ripple, and 20-dB attenuation) was applied after ballistocardiogram artifact correction. Signals were then downsampled to $500 \mathrm{~Hz}$ for further processing.

\section{Brain-machine interface control and feedback}

For the BCI system used in this study, visual stimuli, feature extraction, and classification were all performed using the BCI2000 software platform (73). Participants were asked to perform two motor imagery tasks: imagery of finger-thumb opposition with the left and right hands, and a baseline "rest" task. For the imagery tasks, participants were instructed to use, to the best of their ability, a first-person perspective and kinesthetic rather than visual imagery (74). Participants also overtly practiced the movements before the start of the experiment.

Tasks were cued using visual stimuli (Fig. 2A) that were projected onto a mirror attached to the MRI head coil. For each trial, a rectangular target appeared in the lower left, lower right, or entire bottom portion of the display, which cued the LT, RT, or rest task, respectively. After $1 \mathrm{~s}$, a cursor appeared at the top center of the screen and immediately began falling at a constant rate, such that it would reach the bottom in $4 \mathrm{~s}$. During imagery trials, participants were tasked with using motor imagery to control the horizontal positioning of the cursor so that it would hit the target at the bottom. During rest trials, participants were asked to passively watch the display and refrain from performing the imagery tasks. When the cursor reached the bottom, a 1-s interval ensued, during which the cursor and target either turned yellow in the case of a hit trial or remained unchanged. The next trial began after a 1-s intertrial interval with a blank screen.

Trials were organized into blocks, with each block containing three trials of the same task. A run consisted of 11 pseudorandom permutations of LT and RT blocks interleaved with 12 rest blocks. Each run began and ended with a rest block. The first block of each task was used for classifier calibration and was discarded, leaving a total of $30 \mathrm{LT}, 30 \mathrm{RT}$, and 33 rest trials per run. To evaluate BCI performance, the hit rate was calculated as the number of times the cursor hit the left or right target divided by the number of imagery trials in each run for each participant. The hit rate was calculated over all three runs, and the overall significance was compared with chance $(58 \%, P<0.05$, two-tailed exact binomial test). BCI performance was pooled from three inMRI runs, as no differences in hit rate were found between runs $\left(\mathrm{F}_{(1.9,43.5)}=1.86, P=0.17\right)$.

\section{Feature extraction and cursor control}

After undergoing noise reduction and downsampling, feature extraction and classification were performed to provide BCI control signals (73). Electrodes over the sensorimotor area (F3, F4, C3, C4, Cz, P3, P4, T7, and T8) were re-referenced to large Laplacian derivations for C3 and C4. Spectral amplitudes for $\mathrm{C} 3$ and $\mathrm{C} 4$ were then computed using autoregressive estimation $(75,76)$, with a window length of $500 \mathrm{~ms}$ and bin width of $3 \mathrm{~Hz}$. For all participants, spectral amplitudes were selected from the 9.5-12.5-Hz bin for feature extraction, which allowed the BCI to be controlled with SMR desynchronizations related to motor imagery (43). The fixed 9.5-12.5-Hz bin was selected according to the results of the previous outMRI study showing that it is effective for controlling the BCI (23). The use of a single frequency band also made it easier to analyze and interpret all participants' data as a group. Harmonic noise detected from the MRI scanner precluded the inclusion of beta activity $(13-30 \mathrm{~Hz})$, which can be used for BCI control. 
A control signal for cursor movement was computed from the interhemispheric difference (C4 minus C3) during ERD. At each time point, the control signal was normalized to the zero mean and unit variance based on data from the previous two trials of all three tasks. This normalization provided a linear classifier for the RT and LT tasks. When the C4 spectral amplitude decreased relative to the $\mathrm{C} 3$ spectral amplitude (i.e., was desynchronized), the cursor accelerated to the left. Conversely, sufficient C3 desynchronization resulted in rightward cursor movement.

\section{Statistical Analysis}

\section{fMRI data preprocessing}

All fMRI data preprocessing and analyses were performed using SPM8 (Wellcome Trust Center for Neuroimaging, London, UK). The functional images underwent slice-timing correction and spatial realignment. The realigned images were then normalized to the Montreal Neurological Institute stereotactic space using the standard echo planar imaging template in SPM8. Finally, the normalized images were spatially smoothed using a Gaussian kernel of 6-mm full-width at halfmaximum.

\section{Statistical analyses of fMRI data}

For the first-level analysis, within-subject task effects were examined by including LT, RT, and rest as conditions plus head motion parameters in a general linear model. Onset time and duration of fMRI data corresponded to the 4-s BCI control intervals, during which the cursor was moving and participants were controlling its position. Performance-related effects were examined by including two binary parametric modulators corresponding to the hit and miss trials for the LT and RT tasks.

The second-level analysis revealed greater bilateral dorsal striatum activity during the hit trials than during the miss trials (Fig. 3). PPI analysis was performed to examine functional coupling of the dorsal striatum with other regions throughout the brain (Fig. 4). The dorsal striatum served as a seed region, with the BOLD time series applied as a physiological variable, whereas the parametric modulators from the hit/miss model were used as psychological variables. These psychophysiological variables and their interaction were then applied in a model to identify areas that were functionally coupled with the seed region.

For all designs, data were high-pass filtered (1/128-Hz cutoff) to remove low-frequency drift, and realignment parameters acquired during preprocessing were included to regress out head movement artifacts. Second-level, random-effect model group analyses were then performed using contrast-weighted beta images from the first-level analysis. The height threshold at the voxel level was set to a $P$ value of $<0.001$, and FWE correction at the cluster level $(P<0.05)$ was performed using SPM8's implementation of random field theory.

\section{Striatum parcellation and fMRI}

A map of striatal subdivisions was created using a diffusion-based subcortical gray matter classification technique $(42,77,78)$. In brief, diffusion tensor MR images $\left(b=1,000 \mathrm{~s} / \mathrm{mm}^{2}\right)$ were collected from a separate group (15 volunteers, 5 female, aged $26.7 \pm 10.1$ years), after obtaining written consent and approval by the institutional ethics committee (42). Probabilistic diffusion tractography running between the whole striatum seed and the frontal cortical areas was analyzed using FSL4.1. The cortical subdivision that had the highest connectivity was identified after scaling connectivity in each cortical region relative to the total for each voxel in the entire striatum. The ventral striatal VOI connecting with the orbitofrontal cortex and the motor striatal VOI connecting with M1 and Brodmann area 6, including the SMA and PM, were used. BOLD time series data were extracted from these two striatal VOIs. To assess the time series, data from 
the BCI control periods just after the rest trials were classified into hit and miss trials. In this analysis, data from two participants were excluded $(n=22)$ : one participant had an extreme performance (no miss trials for the selected condition) and the other participant had bumpy BOLD time-series data ( $>2$ SDs from the group data), possibly due to head motion.

\section{LASSO regression analysis of BCI performance}

To predict task performance during RT, LASSO regression (sklearn.linear_model;

http://jupyter.org/) was applied using connectivity with the left motor striatum as an explanatory variable. The LASSO is a linear regression with an L1 norm penalty term (which thus introduces sparseness) (79). A hyperparameter for the penalty weight was determined by 2 -fold cross validation $(\alpha=0.11)$. The explanatory variables were values of connectivity with the left motor striatum, which were calculated from a $10-\mathrm{mm}$ spheric VOI set at the peak coordinate of each cluster in the fMRI analysis. VOIs were set in the following 22 regions: the cerebellum, PM, SMA, AIC, M1, SPL, IPL, dlPFC, LOC, motor striatum, and thalamus (all bilateral). To assess model fitting, the correlation of the determination parameter was calculated $\left(R^{2}=0.87\right)$.

\section{Reference}

1. A. Kübler et al., Patients with ALS can use sensorimotor rhythms to operate a braincomputer interface. Neurology 64, 1775-1777 (2005).

2. M. A. Lebedev, M. A. L. Nicolelis, Brain-machine interfaces: past, present and future. Trends in Neurosciences 29, 536-546 (2006).

3. N. Birbaumer, L. G. Cohen, Brain-computer interfaces: communication and restoration of movement in paralysis. The Journal of physiology 579, 621-636 (2007).

4. S. J. Bensmaia, L. E. Miller, Restoring sensorimotor function through intracortical interfaces: progress and looming challenges. Nat Rev Neurosci 15, 313-325 (2014).

5. E. E. Fetz, Operant conditioning of cortical unit activity. Science 163, 955-958 (1969).

6. K. Shibata, Y. Sasaki, M. Kawato, T. Watanabe, Perceptual learning incepted by decoded fMRI neurofeedback without stimulus presentation. Journal of Vision 12, 282-282 (2012).

7. B. Engelhard, N. Ozeri, Z. Israel, H. Bergman, E. Vaadia, Inducing gamma oscillations and precise spike synchrony by operant conditioning via brain-machine interface. Neuron 77, 361-375 (2013).

8. M. T. deBettencourt, J. D. Cohen, R. F. Lee, K. A. Norman, N. B. Turk-Browne, Closedloop training of attention with real-time brain imaging. Nature Neuroscience 18, 1-9 (2015).

9. A. Ramos-Murguialday et al., Brain-machine interface in chronic stroke rehabilitation: a controlled study. Annals of Neurology 74, 100-108 (2013).

10. F. Pichiorri et al., Brain-computer interface boosts motor imagery practice during stroke recovery. Annals of Neurology 77, 851-865 (2015).

11. K. Shindo et al., Effects of neurofeedback training with an electroencephalogram-based brain-computer interface for hand paralysis in patients with chronic stroke: a preliminary case series study. Journal of Rehabilitation Medicine 43, 951-957 (2011).

12. L. R. Hochberg et al., Reach and grasp by people with tetraplegia using a neurally controlled robotic arm. Nature 485, 372-375 (2012).

13. T. Aflalo et al., Decoding motor imagery from the posterior parietal cortex of a tetraplegic human. Science 348, 906-910 (2015).

14. K. Emmert et al., Meta-analysis of real-time fMRI neurofeedback studies using individual participant data: How is brain regulation mediated? Neuroimage 124, 806-812 (2016).

15. T. Fovet et al., On assessing neurofeedback effects: should double-blind replace neurophysiological mechanisms? Brain 140, e63 (2017). 
16. M. Ninaus et al., Neural substrates of cognitive control under the belief of getting neurofeedback training. Front Hum Neurosci 7, 914 (2013).

17. E. F. Oblak, J. A. Lewis-Peacock, J. S. Sulzer, Self-regulation strategy, feedback timing and hemodynamic properties modulate learning in a simulated fMRI neurofeedback environment. PLoS Comput Biol 13, e1005681 (2017).

18. R. Sitaram et al., Closed-loop brain training: the science of neurofeedback. Nat Rev Neurosci 18, 86-100 (2017).

19. M. A. Lebedev, Cortical ensemble adaptation to represent velocity of an artificial actuator controlled by a brain-machine interface. Journal of Neuroscience 25, 4681-4693 (2005).

20. A. M. Green, J. F. Kalaska, Learning to move machines with the mind. Trends in Neurosciences 34, 61-75 (2011).

21. A. C. Koralek, X. Jin, J. D. Long II, R. M. Costa, J. M. Carmena, Corticostriatal plasticity is necessary for learning intentional neuroprosthetic skills. Nature 483, 331-335 (2012).

22. J. D. Wander et al., Distributed cortical adaptation during learning of a brain-computer interface task. Proceedings of the National Academy of Sciences of the United States of America 110, 10818-10823 (2013).

23. K. Kasahara, C. S. DaSalla, M. Honda, T. Hanakawa, Neuroanatomical correlates of brain-computer interface performance. NeuroImage 110, 95-100 (2015).

24. S. Halder et al., Neural mechanisms of brain-computer interface control. NeuroImage 55, 1779-1790 (2011).

25. B. Blankertz et al., Neurophysiological predictor of SMR-based BCI performance. NeuroImage 51, 1303-1309 (2010).

26. T. Zhang et al., Structural and functional correlates of motor imagery BCI performance: insights from the patterns of fronto-parietal attention network. NeuroImage 134, 475-485 (2016).

27. S. Saha, M. Baumert, Intra- and Inter-subject Variability in EEG-Based Sensorimotor Brain Computer Interface: A Review. Front Comput Neurosci 13, 87 (2019).

28. E. M. Hammer et al., Psychological predictors of SMR-BCI performance. Biol Psychol 89, 80-86 (2012).

29. B. Z. Allison, C. Neuper, in Brain-Computer Interfaces: Applying our Minds to HumanComputer Interaction, D. S. Tan, A. Nijholt, Eds. (Springer London, London, 2010), pp. $35-54$.

30. M. A. Schwemmer et al., Meeting brain-computer interface user performance expectations using a deep neural network decoding framework. Nat Med 24, 1669-1676 (2018).

31. H. E. Schendan, M. M. Searl, R. J. Melrose, C. E. Stern, An FMRI study of the role of the medial temporal lobe in implicit and explicit sequence learning. Neuron 37, 1013-1025 (2003).

32. C. Zich et al., Real-time EEG feedback during simultaneous EEG-fMRI identifies the cortical signature of motor imagery. NeuroImage 114, 438-447 (2015).

33. T. Hinterberger et al., Neuronal mechanisms underlying control of a brain-computer interface. European Journal of Neuroscience 21, 3169-3181 (2005).

34. X. Wan et al., The neural basis of intuitive best next-move generation in board game experts. Science 331, 341-346 (2011).

35. S. Marchesotti et al., Cortical and subcortical mechanisms of brain-machine interfaces. Human Brain Mapping 38, 2971-2989 (2017).

36. G. E. Alexander, M. R. DeLong, P. L. Strick, Parallel organization of functionally segregated circuits linking basal ganglia and cortex. Annu Rev Neurosci 9, 357-381 (1986).

37. S. N. Haber, B. Knutson, The reward circuit: linking primate anatomy and human imaging. Neuropsychopharmacology 35, 4-26 (2010). 
38. M. X. Cohen, J. C. Schoene-Bake, C. E. Elger, B. Weber, Connectivity-based segregation of the human striatum predicts personality characteristics. Nat Neurosci 12, 32-34 (2009).

39. T. Hanakawa, Organizing motor imageries. Neuroscience Research 104, 56-63 (2016).

40. J. D. Salamone, M. Correa, The mysterious motivational functions of mesolimbic dopamine. Neuron 76, 470-485 (2012).

41. K. Wang, D. Smith, M. Delgado, Using fMRI to study reward processing in humans: past, present, and future. Journal of Neurophysiology 115, 1664-1678 (2016).

42. T. Hanakawa, A. M. Goldfine, M. Hallett, A Common Function of Basal Ganglia-Cortical Circuits Subserving Speed in Both Motor and Cognitive Domains. eNeuro 4, (2017).

43. G. Pfurtscheller, F. H. L. Da Silva, Event-related EEG/MEG synchronization and desynchronization: basic principles. Clinical Neurophysiology 110, 1842-1857 (1999).

44. G. E. Alexander, Functional architecture of basal ganglia circuits: neural substrated of parallel processing. Trends in Neurosciences 13, 266-271 (1990).

45. F. A. Middleton, P. L. Strick, Basal ganglia and cerebellar loops. Brain Research Reviews 31, 236-250 (2000).

46. K. Doya, Modulators of decision making. Nature Neuroscience 11, 410-416 (2008).

47. E. Hoshi, L. Tremblay, J. Féger, P. L. Carras, P. L. Strick, The cerebellum communicates with the basal ganglia. Nature neuroscience 8, 1491-1493 (2005).

48. A. C. Bostan, R. P. Dum, P. L. Strick, The basal ganglia communicate with the cerebellum. Proceedings of the National Academy of Sciences of the United States of America 107, 8452-8456 (2010).

49. S. Halder et al., Prediction of brain-computer interface aptitude from individual brain structure. Frontiers in human neuroscience 7, 105 (2013).

50. C. Zich, M. De Vos, C. Kranczioch, S. Debener, Wireless EEG with individualized channel layout enables efficient motor imagery training. Clinical Neurophysiology 126, 698-710 (2015).

51. Y. Zhang, G. Zhou, J. Jin, X. Wang, A. Cichocki, Optimizing spatial patterns with sparse filter bands for motor-imagery based brain-computer interface. Journal of Neuroscience Methods 255, 85-91 (2015).

52. T. Hanakawa et al., Functional properties of brain areas associated with motor execution and imagery. J Neurophysiol 89, 989-1002 (2003).

53. T. Hanakawa, M. A. Dimyan, M. Hallett, Motor planning, imagery, and execution in the distributed motor network: a time-course study with functional MRI. Cereb Cortex 18, 2775-2788 (2008).

54. M. J. Hayashi et al., Hemispheric asymmetry of frequency-dependent suppression in the ipsilateral primary motor cortex during finger movement: a functional magnetic resonance imaging study. Cerebral Cortex 18, 2932-2940 (2008).

55. K. Foerde, D. Shohamy, Feedback timing modulates brain systems for learning in humans. The Journal of Neuroscience 31, 13157-13167 (2011).

56. M. Sawada et al., Function of the nucleus accumbens in motor control during recovery after spinal cord injury. Science 350, 98-101 (2015).

57. J. R. Wolpaw, D. J. McFarland, Control of a two-dimensional movement signal by a noninvasive brain-computer interface in humans. Proceedings of the National Academy of Sciences of the United States of America 101, 17849-17854 (2004).

58. C. Sannelli, C. Vidaurre, K. R. Muller, B. Blankertz, A large scale screening study with a SMR-based BCI: Categorization of BCI users and differences in their SMR activity. PLoS One 14, e0207351 (2019).

59. H. H. Yin, B. J. Knowlton, The role of the basal ganglia in habit formation. Nat Rev Neurosci 7, 464-476 (2006). 
60. X. Wan et al., Developing intuition: neural correlates correlates of cognitive-skill learning in caudate nucleus. The Journal of Neuroscience 32, 17492-17501 (2012).

61. M. Haruno, M. Kimura, C. D. Frith, Activity in the nucleus accumbens and amygdala underlies individual differences in prosocial and individualistic economic choices. Journal of cognitive neuroscience 26, 1861-1870 (2014).

62. J. Crinion et al., Language control in the bilingual brain. Science 312, 1537-1540 (2006).

63. R. Cools, R. B. Ivry, M. D'Esposito, The human striatum is necessary for responding to changes in stimulus relevance. J Cogn Neurosci 18, 1973-1983 (2006).

64. F. Bacigalupo, S. J. Luck, Lateralized Suppression of Alpha-Band EEG Activity As a Mechanism of Target Processing. J Neurosci 39, 900-917 (2019).

65. W. Klimesch, alpha-band oscillations, attention, and controlled access to stored information. Trends Cogn Sci 16, 606-617 (2012).

66. J. Lauwereyns, K. Watanabe, B. Coe, O. Hikosaka, A neural correlate of response bias in monkey caudate nucleus. Nature 418, 413-417 (2002).

67. M. Corbetta, G. L. Shulman, Control of goal-directed and stimulus-driven attention in the brain. Nat Rev Neurosci 3, 201-215 (2002).

68. R. C. Oldfield, The assessment and analysis of handedness: the Edinburgh inventory. Neuropsychologia 9, 97-113 (1971).

69. K. Omata, T. Hanakawa, M. Morimoto, M. Honda, Spontaneous slow fluctuation of eeg alpha rhythm reflects activity in deep-brain structures: a simultaneous EEG-fMRI study. PLoS ONE 8, e66869 (2013).

70. P. J. Allen, G. Polizzi, K. Krakow, D. R. Fish, L. Lemieux, Identification of EEG events in the MR scanner: the problem of pulse artifact and a method for its subtraction.

NeuroImage 8, 229-239 (1998).

71. J. R. Ives, S. Warach, F. Schmitt, R. R. Edelman, D. L. Schomer, Monitoring the patient's EEG during echo planar MRI. Electroencephalography and Clinical Neurophysiology 87, 417-420 (1993).

72. C. G. Bénar et al., Quality of EEG in simultaneous EEG-fMRI for epilepsy. Clinical Neurophysiology 114, 569-580 (2003).

73. G. Schalk, D. J. McFarland, T. Hinterberger, N. Birbaumer, J. R. Wolpaw, BCI2000: A general-purpose brain-computer interface (BCI) system. IEEE Transactions on Biomedical Engineering 51, 1034-1043 (2004).

74. C. Neuper, R. Scherer, M. Reiner, G. Pfurtscheller, Imagery of motor actions: Differential effects of kinesthetic and visual-motor mode of imagery in single-trial EEG. Cognitive Brain Research 25, 668-677 (2005).

75. S. L. Marple, Digital spectral analysis with applications. Englewood Cliffs, NJ, PrenticeHall, Inc., 1987, 512 p. 1, (1987).

76. D. J. McFarland, A. T. Lefkowicz, J. R. Wolpaw, Design and operation of an EEG-based brain-computer interface with digital signal processing technology. Behavior Research Methods, Instruments, \& Computers 29, 337-345 (1997).

77. T. E. J. Behrens et al., Characterization and propagation of uncertainty in diffusionweighted MR imaging. Magnetic Resonance in Medicine 50, 1077-1088 (2003).

78. A. C. Tziortzi et al., Connectivity-based functional analysis of dopamine release in the striatum using diffusion-weighted MRI and positron emission tomography. Cerebral Cortex, bhs397 (2013).

79. R. Tibshirani, Regression shrinkage and selection via the Lasso. J Roy Stat Soc B Met 58, 267-288 (1996).

80. C. R. Hall, K. A. Martin, Measuring movement imagery abilities: A revision of the Movement Imagery Questionnaire. Journal of Mental Imagery 21, 143-154 (1997). 


\section{Funding:}

Japan Agency for Medical Research and Development grants 19dm0207070s0001 and

19dm0307003h0002 (TH)

Japan Society for the Promotion of Science grants KAKANHI 19H05726 and 19H03536 (TH) and

KAKANHI 20H04236 (KK)

Japan Science and Technology Agency grant FORESTO JPMJFR206G (KK)

Charles S. DaSalla is currently affiliated with the Office of Research and Innovation, Tokyo Institute of

Technology.

\section{Author contributions:}

Conceptualization: $\mathrm{TH}$

Methodology: KK, CSD, MH, and TH

Investigation: $\mathrm{KK}$ and $\mathrm{CSD}$

Writing - original draft: KK, CSD, and TH

Writing - review \& editing: $\mathrm{KK}, \mathrm{CSD}, \mathrm{MH}$, and $\mathrm{TH}$

Funding acquisition: TH, KK

Resources: $\mathrm{MH}$ and $\mathrm{TH}$

Supervision: $\mathrm{MH}$ and $\mathrm{TH}$

Competing interests: Authors declare that they have no competing interests.

Data and materials availability: All data needed to evaluate the conclusions in the paper are present in the paper and/or the Supplementary Materials. Additional data related to this paper may be requested from the authors.

\section{Figures}

\section{A}

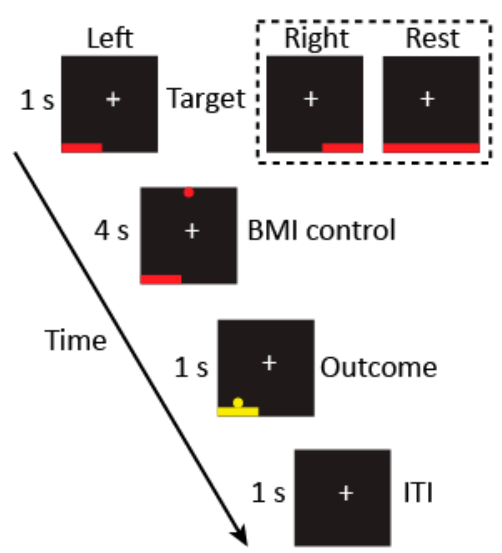

B

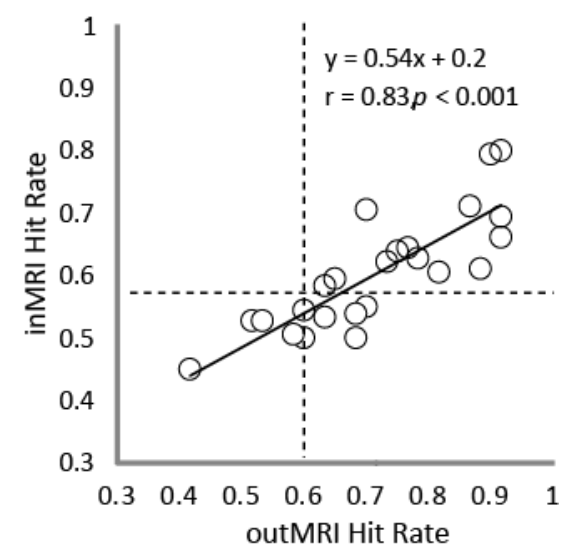

Fig. 1. Experimental design and correlation between hit rates for sessions outside and inside MRI. (A) An example of a "left" trial, with "right" and "rest" targets shown in the dashed box. ITI, intertrial interval. (B) Hit rates for sessions outside the MRI (outMRI) are plotted against those inside the MRI (inMRI) for each participant. On average, the inMRI hit rate (mean $\pm \mathrm{SD}, 0.60 \pm 0.09$ ) was significantly lower than the outMRI hit rate $(0.72 \pm 0.14)$, as determined using a paired $t$ test $\left(\mathrm{t}_{(23)}=6.9, P<0.001\right)$. However, hit rates were strongly correlated between the inMRI and outMRI sessions $(r=0.83, P<0.001)$. Dashed lines indicate the minimum significant hit rate (out of 60 trials per task for outMRI and 90 trials per task for inMRI; $P<0.05$ for both). Solid line indicates the linear regression. 

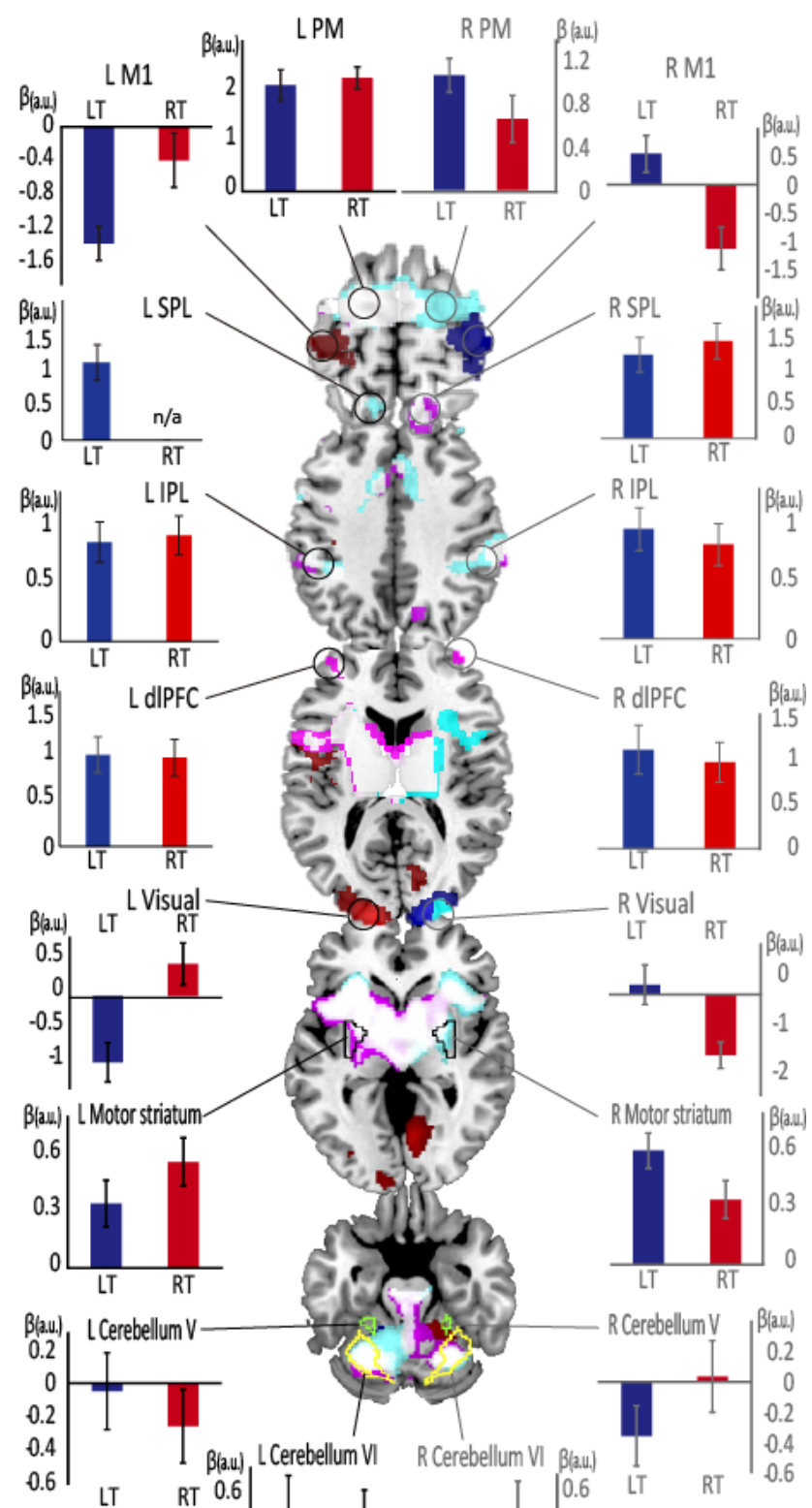

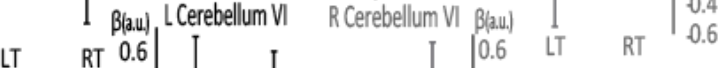

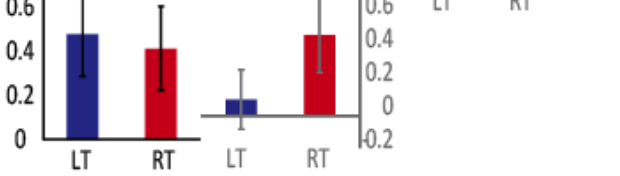

Fig. 2. Task-related signal changes for the BCI task compared with signals at rest. Red and blue areas denote BCI-related activity during right target (RT) and left target (LT) tasks, respectively, which differed significantly when comparing the two tasks. Magenta- and cyan-colored areas represent nonlateralized activity during RT and LT tasks, respectively, and white areas represent their overlap. PM premotor area; M1, primary motor area; SPL, superior parietal lobule; IPL, inferior parietal lobule; dIPFC, dorsolateral prefrontal cortex; a.u., arbitrary units. 
A

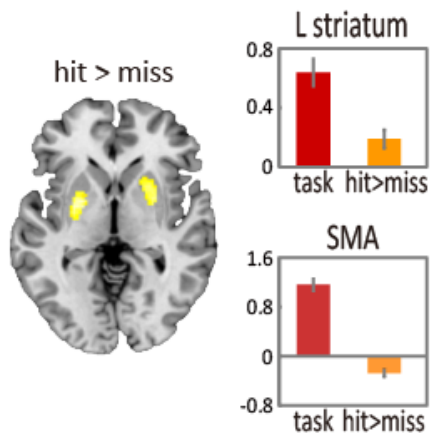

B

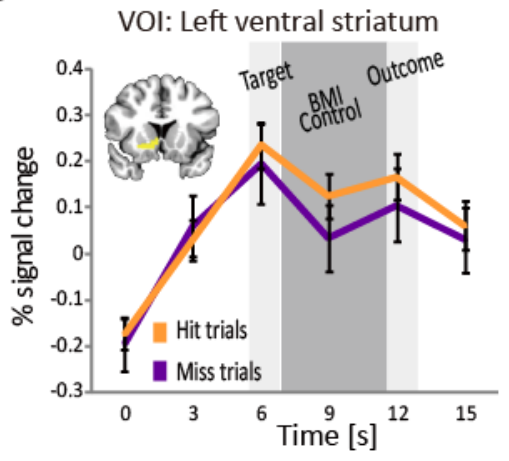

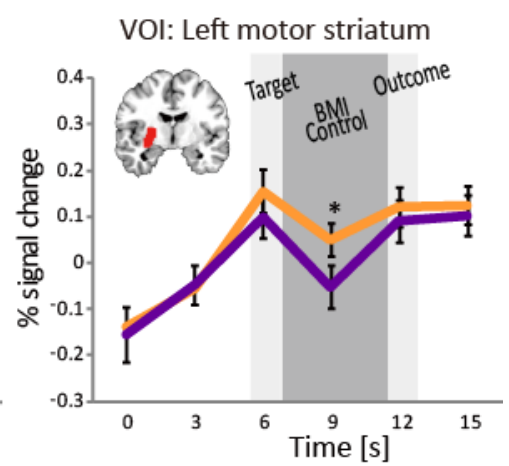

Fig. 3. Areas of greater activation during the hit trials than during the miss trials. (A) Only the bilateral dorsal striatum (yellow) showed greater BCI-related activity during the hit trials than during the miss trials $(P<0.05$ cluster-level FWE corrected). Supplementary motor areas (SMA) showed greater BCI-related activity during the miss trials than during the hit trials. (B) Signal change time courses for the left ventral and left motor striatum. Both the ventral and motor striatum, defined by diffusion MRI, showed BCI task-related activity. However, only motor striatum exhibited greater activity for the hit trials than for the miss trials during the $\mathrm{BCI}$ control period (dark gray shaded areas, which lag $6 \mathrm{~s}$ behind the actual BCI control period to accommodate the hemodynamic delay).

A

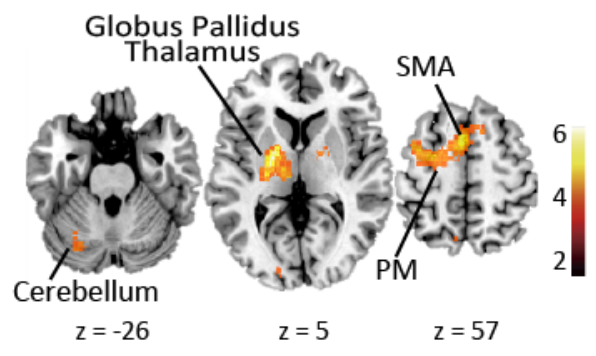

B

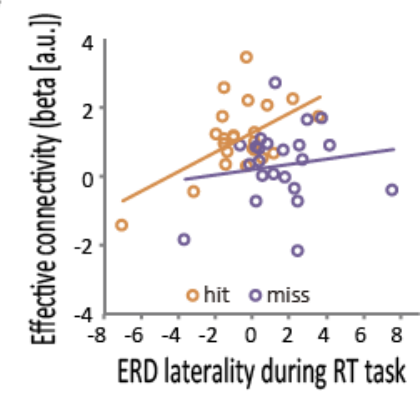

Fig. 4. Motor cortex-basal ganglia connectivity correlates with successful BCI control. (A) Hit-related effective connectivity with the left motor striatum increased in the cerebellum, globus pallidus, thalamus, and cortical motor areas, including the supplementary motor area (SMA) and dorsal premotor cortex (PM). (B) Effective connectivity between the motor striatum and SMA correlated with the laterality of event-related desynchronization (ERD) during the hit trials $(r=0.56, P=0.005)$ but not during the miss trials $(r=0.15, P$ $=0.497)$ of the right-target $(\mathrm{RT})$ task. Each circle represents data from one individual. 
A

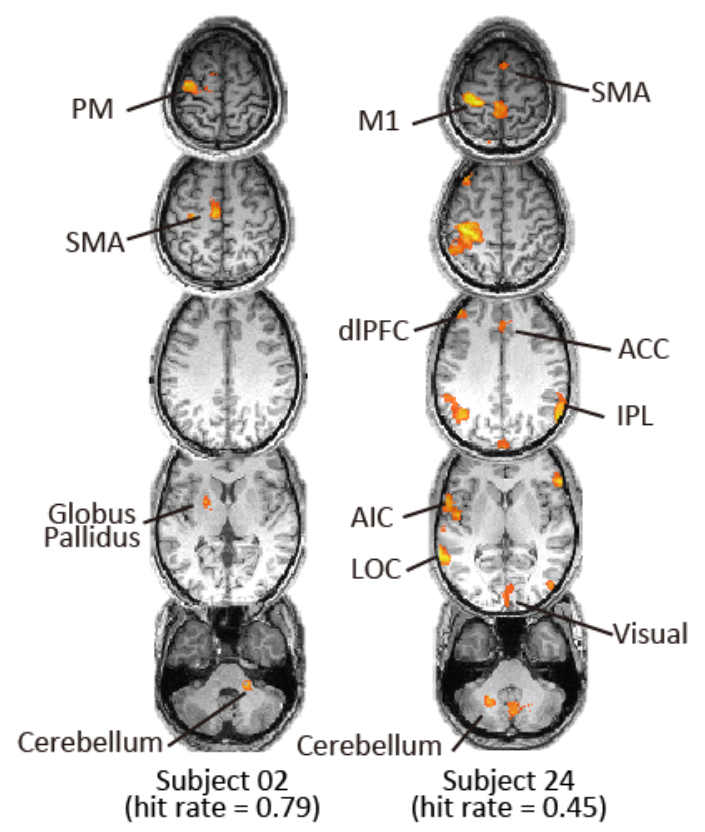

B

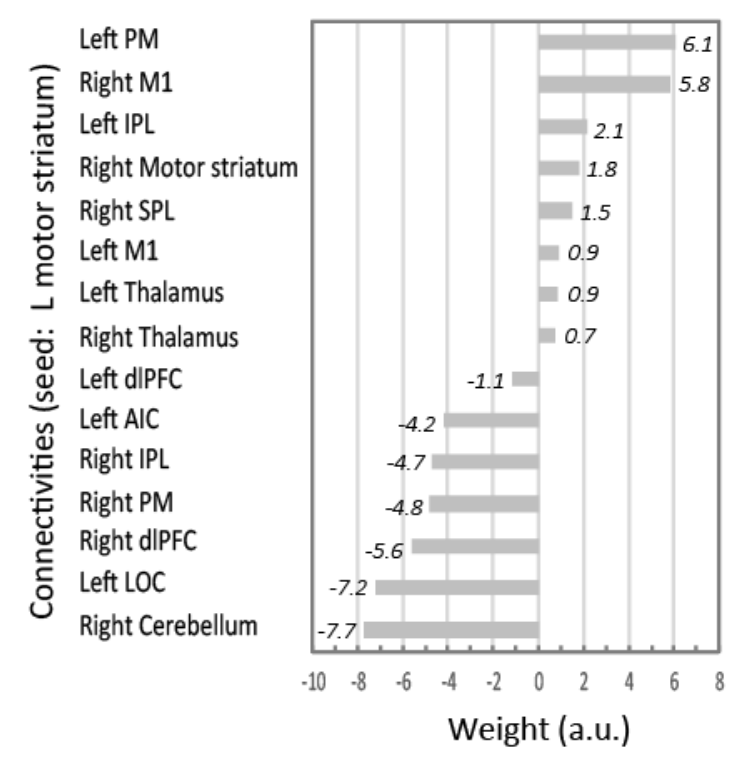

Fig. 5. Effective connectivity with dorsal striatum represents individual differences in BCI performance. (A) Effective connectivity analysis in two representative participants. Subject 02 , who had good BCI performance (hit rate of 0.79 ), showed $\mathrm{BgCN}$ connectivity only, whereas subject 24, who had poor BCI performance (hit rate of 0.45 ), showed NfCN connectivity in addition to $\mathrm{BgCN}$ connectivity. (B) The LASSO regression revealed that good $\mathrm{BCI}$ performance correlated with $\mathrm{BgCN}$ connectivity, whereas it was negatively affected by connectivity with $\mathrm{NfCN}$, including the lateral occipital cortex (LOC), anterior insula cortex (AIC), dorsolateral prefrontal cortex (dlPFC), and inferior parietal lobule (IPL). Weights of the left IPL, M1, dlPFC, right motor striatum, superior parietal lobule (SPL), and bilateral thalamus were less than 3.0, which represents a weak correlation. 